\title{
Phonotype: a New Taxonomy for mHealth Research
}

\author{
Bruce L. Rollman, MD, MPH ${ }^{1,2}$ (1) and David A. Brent, $\mathrm{MD}^{3}$
}

'Division of General Internal Medicine, Department of Medicine, University of Pittsburgh School of Medicine, Pittsburgh, PA, USA; ${ }^{2}$ Center for Behavioral Health and Smart Technology, University of Pittsburgh School of Medicine, Pittsburgh, PA, USA; ${ }^{3}$ Division of Child \& Adolescent Psychiatry, Department of Psychiatry, University of Pittsburgh School of Medicine, Pittsburgh, PA, USA.

J Gen Intern Med 35(6):1881-3

DOI: $10.1007 / \mathrm{s} 11606-019-05407-7$

(c) Society of General Internal Medicine 2019

Our phones and computers have become reflections of our personalities, our interests, and our identities. They hold much that is important to us. ${ }^{1}$

James B. Comey, Former FBI Director

Genotype and phenotype are established terms used to describe the set of genes that are unique to a person's genetic makeup, and the observable characteristics resulting from the interaction of that genotype with the environment, respectively. Adding to this taxonomy, we propose a new term, "phonotype," to describe how information collected by an individual's smartphone can be utilized to understand their behavior and promote health.

While similar phrases including reality mining, digital phenotyping, and personal informatics have also been used to describe research involving smartphone-collected data, ${ }^{2}$ we advocate for the term "phonotype" instead given its (1) clear connotation to smartphones; (2) "-type" suffix consistency with genotype and phenotype; and (3) single-word simplicity. Moreover, the term captures the potential use of data obtained from smartphones to characterize human activity and behavior in ways that can be mapped onto one's genotype and phenotype.

\section{A NEW ERA IN PERSONALIZED MEDICINE}

Internet-connected smartphones began to appear on the consumer market in the early 2000s, and have become nearly ubiquitous in the decade following the launch of the first-generation Apple iPhone in 2007 and release of Google's Android operating system approximately 1 year later. It is now estimated there are over 2.5 billion smartphones in use worldwide with high rates of

Twitter handle @HealthTechPitt to Center for Behavioral Health and Smart Technology

Received August 15, 2019

Revised August 15, 2019

Accepted September 20, 2019

Published online November 8, 2019 ownership in both advanced and emerging economies. ${ }^{3}$ Furthermore, they have become an essential component in the lives of most Americans with over $80 \%$ of US adults owning a device and ownership rates that are similar by race and high even among the less affluent (71\% of individuals with annual incomes $\leq$ $\$ 30,000$ own a smartphone). ${ }^{4}$

\section{PHONOTYPE DATA LEVELS}

Unlike earlier generations of mobile phones, modern smartphones contain accelerometers, gyroscopes, light sensors, microphones, cameras, and GPS. These sensors passively collect "digital exhaust" ${ }^{2}$ that can be analyzed to establish normal baseline and diurnal patterns in how an individual smartphone user types, taps, speaks, moves, sleeps, and uses their apps (phonotype level 1). Moreover, this information can be augmented with passively collected smartphone data on social interaction (e.g., call logs, text messages, emails, address books, photographs, and social media posts) and media exposure (e.g., news, podcasts, videos, music, and web browsing) to generate novel insights into an individual's state of mind and social ecology (phonotype level 2).

Further enhancing the personalization of one's phonotype, many individuals, particularly those living with a chronic medical condition, will "actively" upload their biometric data to their smartphones via a Bluetooth-connected heart rate monitor, blood pressure cuff, scale, glucometer, electrocardiograph, or smartwatch that are widely available (phonotype level 3). Lastly, we anticipate electronic medical record (EMR) systems will soon automatically "push" clinical data to a patient's smartphone (e.g., weight, vital signs, medications prescribed, laboratory results, and health services utilization (phonotype level 4)), thereby informing delivery of even more personalized interventions, while becoming capable of downloading smartphone-collected data for presentation to clinicians in an organized format (phonotype levels 1-3).

\section{PHONOTYPE STRATA}

Sophisticated machine learning and artificial intelligence algorithms are increasingly able to analyze the enormous amounts of data collected on smartphones and then classify 
users into phonotype strata. Similar to the "Health Predisposition Reports" created by 23andMe and other personalized genomics firms, we anticipate these reports will present easily interpretable risk probabilities that the user will develop certain conditions (e.g., depression, suicidal ideations) and estimate their control of recognized disorders (Table 1). Yet unlike "fixed" Health Predisposition Reports informed by genetic data, these algorithms will regularly update a patient's phonotype strata in response to changes in their behavior, circumstances, and other information collected by their smartphone.

\section{PHONOTYPE APPLICATIONS TO IMPROVE CLINICAL CARE}

To date, the most studied phonotypes have related to mental health disorders, ${ }^{2,5}$ but many other behavioral patterns can be analyzed that, in conjunction with a clinical examination and select testing, may help clinicians identify the correct diagnosis, initiate treatment, and then monitor their patient's response between face-to-face visits. Some of these patterns include:

- Changes in word choice and tone, social media content, emojis, and websites visited which may be a warning for imminent suicidal risk, depression relapse, schizophrenia, or another mental health condition. ${ }^{5}$

- Smartphone use during normal sleeping hours which could be related to anxiety, mania, heart failure, or the presence of another chronic illness.

- Changes in how quickly the user types and errors made combined with GPS data on physical activity that could be early signs of an adverse drug event, dementia, intoxication, or a visual disturbance.

- Increases in physical activity, sleep, and concentration may indicate improved control of depression, pain, remission of substance use disorder, or sleep apnea.

We foresee that the software algorithms used to categorize individuals into phonotype strata will also generate a variety of highly personalized, evidence-based, and smartphone-

Table 1 A Sample Report Portraying Personalized Health Predisposition and Disease Control by Phonotype Strata

\begin{tabular}{|c|c|c|c|c|c|c|}
\hline \multirow[b]{3}{*}{ Phenotype } & \multicolumn{6}{|c|}{ Phonotype strata } \\
\hline & \multicolumn{3}{|c|}{ Health predisposition } & \multicolumn{3}{|c|}{ Disease control } \\
\hline & $\begin{array}{l}\text { Low } \\
\text { risk }\end{array}$ & $\begin{array}{l}\text { Elevated } \\
\text { risk }\end{array}$ & $\begin{array}{l}\text { Pre- } \\
\text { disease }\end{array}$ & Good & Fair & Poor \\
\hline Addiction & - & & & & & \\
\hline Dementia & - & & & & & \\
\hline Depression & & $\bullet$ & & & & \\
\hline Diabetes & & & & $\bullet$ & & \\
\hline Heart failure & & $\bullet$ & & & & \\
\hline Hypertension & & & & $\bullet$ & & \\
\hline Insomnia & & & & $\bullet$ & & \\
\hline Pain & & & & & $\bullet$ & \\
\hline Suicidal & & $\bullet$ & & & & \\
\hline ideation & & & & & & \\
\hline
\end{tabular}

delivered just-in-time adaptive interventions (JITAIs) for patients to consider. ${ }^{6}$ When these computer-generated recommendations also advise the start or adjustment of a medication or a diagnostic test, we anticipate they will first be routed to the patient's physician via EMR for their review and approval first. Then, depending upon the urgency and complexity of the situation (e.g., potential suicidal ideations, substance abuse relapse), the physician or a practice nurse will contact the patient via smartphone or request an office visit to discuss this advice with follow-up provided by care managers, virtual medical coaches, chatbots, home care visits, and other means.

\section{CONCLUSIONS}

High rates of smartphone ownership have led to the rapid and unobtrusive accumulation of enormous amounts of personal- and population-level data that can be analyzed in real time and may finally help realize the promise of personalized medicine. We advocate for the term "phonotype" to describe how smartphone-collected data may be used to improve health, and suggest several terms in an effort to provide a common taxonomy that may advance mHealth research. Further study is needed to (1) validate phonotype strata to the risk and presence of specific behavioral and medical conditions; (2) understand how differences in culture, lifestyle, age, gender, and environment may affect phonotype; (3) balance patients' data privacy concerns, particularly when life-threatening events may be present ${ }^{7}$; and (4) demonstrate the effectiveness and cost-effectiveness of just-in-time interventions that are targeted to specific phonotype strata.

Acknowledgments: We thank David C. Mohr for his helpful feedback on a draft of this paper.

Corresponding Author: Bruce L. Rollman, MD, MPH; Division of General Internal Medicine, Department of Medicine, University of Pittsburgh School of Medicine, Pittsburgh, PA, USA (e-mail: rollmanbl@upmc.edu).

Funding Information This work is supported by a grant from the National Institute of Mental Health (P5OMH115838).

\section{Compliance with Ethical Standards:}

The opinions and content expressed are solely the responsibility of the authors and do not necessarily represent the official views of our funding organization.

Conflict of Interest: The authors declare that they do not have a conflict of interest.

\section{REFERENCES}

1. Comey JB. New Technologies Should Not Hinder Law Enforcement Surveillance. In: Merino N, ed. Domestic Surveillance. Farmington Hills, MI: Greenhaven Press, Reprint Edition; 2015. 
2. Mohr DC, Zhang M, Schueller SM. Personal Sensing: Understanding Mental Health Using Ubiquitous Sensors and Machine Learning. Annu Rev Clin Psychol 2017;13:23-47.

3. Taylor K, Silver L. Smartphone Ownership Is Growing Rapidly Around the World, but Not Always Equally. Global Attitudes \& Trends Web site Published 2019. Updated February 5, 2019. Accessed August 12, 2019

4. Mobile Fact Sheet. Pew Research Center. Internet \& Technology Web site. https://www.pewinternet.org/fact-sheet/mobile/. Published 2019. Accessed August 12, 2019

5. Torous J, Larsen ME, Depp C, et al. Smartphones, Sensors, and Machine Learning to Advance Real-Time Prediction and Interventions for Suicide Prevention: a Review of Current Progress and Next Steps. Curr Psychiatry Rep 2018;20:51.

6. Nahum-Shani I, Smith SN, Spring BJ, et al. Just-in-Time Adaptive Interventions (JITAIs) in Mobile Health: Key Components and Design
Principles for Ongoing Health Behavior Support. Ann Behav Med 2017;52:446-462.

7. Allen NB, Nelson BW, Brent D, Auerbach RP. Short-term prediction of suicidal thoughts and behaviors in adolescents: Can recent developments in technology and computational science provide a breakthrough? $J$ Affect Disord 2019;250:163-169.

Publisher's Note Springer Nature remains neutral with regard to jurisdictional claims in published maps and institutional affiliations. 